\title{
ON THE APPLICABILITY OF THE CONCEPT OF CONTROL WAVE PROCESS TO THE $B 2 \rightarrow R$ MARTENSITIC TRANSFORMATION IN B2 TITANIUM NICKELIDE ALLOYS
}

\author{
M. P. Kashchenko ${ }^{1,2}$ and V. G. Chashchina ${ }^{1,2}$
}

UDC 669.018 .2

Within the framework of the dynamic theory based on the synthesis of concepts of heterogeneous nucleation and wave growth of martensite crystals, the possibility of description of morphological parameters during the $B 2 \rightarrow R$ transformation is analyzed. It is demonstrated that the calculated and observed orientations of the habit planes can be matched. The calculated orientation relationships are given and the results obtained are discussed.

Keywords: dynamic theory, martensitic transformations, titanium nickelide, morphological parameters.

\section{INTRODUCTION}

Nowadays there are strong reasons to believe that the dynamic theory [1-4] of reconstructive martensitic transformations (MT) adequately describes a wide number of the observed peculiarities of cooperative structure reorganization. Such transformations possess evident characteristics of the first-order transitions, proceed with noticeable deviation of phases from the equilibrium temperature, and involve the limiting case of supersonic crystal growth. Naturally, the question arises in what measure this dynamic theory is applicable to a description of transformations with less evident characteristics of the first-order transitions. In this regard, we note that in titanium nickelide and alloys on its basis, three MT variants $\left(B 2 \rightarrow B 19, B 2 \rightarrow R\right.$, and $\left.B 2 \rightarrow B 19^{\prime}\right)$ are often observed [5, 6]. From them the $B 2 \rightarrow B 19$ MT involves the maximum change of the relative volume, and the $B 2 \rightarrow R$ MT involves the minimum change of the relative volume. In $[7,8]$ it was demonstrated that methodology suggested in [1-4] provides a reasonable description of the habit planes of the $B 2 \rightarrow B 19$ MT for which it is suffice to consider the threshold regime of propagation of the control wave process (CWP). Recall that the concept of the initial excited state (IES) arising in the elastic field of a dislocation nucleation center (DNC) plays the key role in the dynamic theory [1-4]. The oscillatory character of the IES creates the CWP transferring the threshold strain that breaks the stability of the initial phase. The concepts of the heterogeneous nucleation and wave growth are synthesized if we consider that the wave normals $\boldsymbol{n}_{\mathbf{1}}$ and $\boldsymbol{n}_{2}$ of the wave beams forming the CWP and describing tension $\left(\varepsilon_{1}>0\right)$ and compression $\left(\varepsilon_{2}<0\right)$ strains in the superposition region are collinear to the eigenvectors $\xi_{i}(i=1,2)$ of the strain tensor of the elastic field of the defect in the nucleation region:

$$
\boldsymbol{n}_{1}|| \xi_{1}, \boldsymbol{n}_{2}|| \xi_{2}, \boldsymbol{n}_{1} \perp \boldsymbol{n}_{2},\left|n_{i}\right|=\left|\xi_{i}\right|=1 .
$$

The normal $\boldsymbol{N}_{\mathrm{w}}$ to the habit plane determined by the CWP propagation direction is given by the expression

$$
\boldsymbol{N}_{\mathrm{w}} \| \boldsymbol{n}_{2}-\boldsymbol{n}_{1} \mathfrak{x}, \mathfrak{x}=\frac{v_{2}}{v_{1}}
$$

${ }^{1}$ Ural Federal University Named after the First President of Russia B. N. Eltsin, Ekaterinburg, Russia; ${ }^{2}$ Ural State Forest Engineering University, Ekaterinburg, Russia, e-mail: mpk46@mail.ru. Translated from Izvestiya Vysshikh Uchebnykh Zavedenii, Fizika, No. 7, pp. 76-81, July, 2013. Original article submitted April 10, 2013. 
where $v_{1}$ and $v_{2}$ are the moduli of the propagation velocities of waves in the directions $\boldsymbol{n}_{1}$ and $\boldsymbol{n}_{2}$. At low threshold strains $\varepsilon_{\text {th }}$, the relationship

$$
æ=\frac{v_{2}}{v_{1}}=k \approx \sqrt{\frac{\varepsilon_{1}}{\left|\varepsilon_{2}\right|}}
$$

holds true. To describe correctly the orientation relations (OR), transition from the threshold to final strains is required. It can be demonstrated that for the $B 2 \rightarrow B 19$ MT and the CWP variant $[9,10]$ providing the fastest transformation of the $\{110\}_{B 2}$ planes, the OR can be calculated by analogy with [10]. Here from the same positions we study the $B 2 \rightarrow R$ MT as the first-order transition, but close to the second-order transition, because the threshold strain, the temperature hysteresis between the direct and reverse transformation, and the thermal effect are small. Various aspects of the problem of the $B 2 \rightarrow R$ transformation have already been analyzed in Chapter 3 of [5] and in [6,11]. For this reason, here we present only the information required for conclusions derived within the framework of the dynamic theory of forming martensite crystals. Arguments (and counterarguments) in favor of the CWP concept regarding the $B 2 \rightarrow R$ transition are stated as required.

\section{TENSION AND COMPRESSION STRAIN RATIO}

The evolution of the $B 2 \rightarrow R$ instability channel is determined by the observed tension strain $\varepsilon^{\|}$of the lattice along one of the symmetry axes $\left\langle 111>_{B 2}\right.$ and by the compression strain $\varepsilon^{\perp}$ in the transverse directions. Values of the final strains in the vicinity of $M_{\mathrm{s}}$ are much smaller in comparison with the $B 2 \rightarrow B 19$ reorganization. Thus, for example, accurate measurements for the aged $\mathrm{Ti}_{49} \mathrm{Ni}_{51}$ alloy gave $\varepsilon^{\|} \approx 1.1 \%$ and $\varepsilon^{\perp} \approx-0.56 \%$.

We first assume that the CWP creates the final tension strain $\varepsilon_{1}$ along the $\langle 111\rangle_{B 2}$ direction and the compression strain $\varepsilon_{2}$ along one of the transverse directions (for example, $<1 \overline{2} 1>_{B 2}$ ). Then under conditions of low elastic anisotropy, the strain ratio created by the CWP will be $\left|\varepsilon_{1} / \varepsilon_{2}\right| \approx \mathfrak{x}^{-2} \approx 1$. Therefore, the observed ratio $\left|\varepsilon^{\|} / \varepsilon^{\perp}\right| \approx 2$ indicated above cannot be produced directly by the CWP of the chosen type. It seems likely that the final resultant strain contains additional contribution caused by the loss of stability according to an unfinished soft-mode scenario (as indicated above, the $B 2 \rightarrow R$ transition is close to the second-order transition). However, here we focus our attention on a less widespread variant of control in which the main role is played by the CWP transferring the threshold strain without tension along $\langle 111\rangle_{B 2}$.

In this case, it is natural to consider that the CWP induces the fastest compression of the plane orthogonal to $<111>_{B 2}$. The condition $\left|\varepsilon^{\|} / \varepsilon^{\perp}\right| \approx 2$ and the relationship $\left|\varepsilon_{1}^{\perp} / \varepsilon_{2}^{\perp}\right| \approx \mathfrak{x}^{-2} \approx 1$ hold both true and are not contradictory. Moreover, the dynamic mechanism can play the key role, because the compression it induces will create quasistatic tension in the direction orthogonal to the compression plane. Then the lattice tension is an indirect consequence of the CWP. Indeed, within the framework of the linear elasticity theory of an isotropic medium, the uniaxial tension $\varepsilon^{\|}$is expressed in terms of the transverse isotropic compression strain $\varepsilon^{\perp}$ as follows:

$$
\varepsilon^{\|}=2 v(1-v)^{-1} \varepsilon^{\perp} .
$$

Then, determining the Poisson coefficient

$$
v=0.5\left[1-0.5\left(C_{11}-C_{12}\right) /\left(C_{11}+C_{12}\right)\right] \text {, }
$$

we can estimate $\varepsilon^{\|}$considering that the CWP induces the observed strain $\varepsilon^{\perp}$. Using the data shown in Fig. 2.6 of [5] (for $\mathrm{Ti}_{49} \mathrm{Ni}_{51}$ single crystals cooled in a furnace) and assuming that $C_{11}$ values for $\mathrm{Ti}_{49} \mathrm{Ni}_{51}$ and $\mathrm{Ti}_{50} \mathrm{Ni}_{50}$ are close, for the elastic moduli (in GPa) we obtain: 


$$
C_{11} \approx 155, C_{12} \approx 125, C_{44} \approx 26
$$

From Eqs. (6) and (5) we obtain $v \approx 0.4732$. Then for $\varepsilon^{\perp} \approx 0.56 \%$ and for the $v$ value calculated from Eq. (4) we obtain $\varepsilon^{\|} \approx 1.006 \%$. Though this estimate is smaller than the observed value equal to $1.1 \%$, it can be accepted as quite satisfactory with allowance for errors of the employed data and linearity of the employed theory.

We note that the presence of strains of one sign in the CWP is not an obstacle for the CWP application, because at small strains (for the first-order transitions close to the second-order transitions), the role of the habit plane favorable for minimization of elastic distortion energy at the interface between the co-existing phases is not determining. An analysis of the morphological parameters can provide additional arguments for choosing among different CWP variants.

\section{VARIANTS OF THE HABIT PLANES}

The habit planes of the $R$-martensite crystals are close to $\{110\}_{B 2}$. Unfortunately, the degree of proximity was not indicated in [5]. Therefore, assuming as previously that the habit planes are determined by the CWP, we now consider their different variants.

In the approximation of the isotropic elastic properties, the pairs of the wave normals can be matched especially simply with the observed habit planes, since in this case we obviate the need for calculating the parameter $æ=1$. For a preset orientation of the dislocation line $\Lambda$, we can always find a pair of wave normals (that together with $\Lambda$ form three orthogonal vectors) which describes, according to Eq. (2), any of the family of habit planes parallel to $\Lambda$. If the plane is parallel to $\Lambda$, the line of its intersection with the plane perpendicular to $\Lambda$ must be determined first. Then orientations of the normals $\boldsymbol{n}_{1}$ and $\boldsymbol{n}_{2}$ are determined by rotating the intersection line through the angles $\pm \pi / 4$ about the $\Lambda$ axis. Though the analysis in the preceding section has allowed us to limit variants of orientations $\Lambda$, for a comparison we now consider typical set of orientations corresponding to the symmetry axes of the cubic lattice.

1. Let we have $\Lambda \|<10 \overline{1}>_{B 2}$.

1.1. The wave beams transferring the tension strain in the $\left\langle\overline{1} 1 \overline{1}>_{B 2}\right.$ directions and the compression strain in the $<121>_{B 2}$ orthogonal directions are excited with wave normals $\boldsymbol{n}_{1} \|<\overline{1} 1 \overline{1}_{B 2}$ and $\boldsymbol{n}_{2} \|<121>_{B 2}$. Then from Eq. (2) for $æ \approx 0.9897 \approx 1$ we obtain the habit planes almost coinciding with $\{414\}_{B 2}$ and having the least angles of $\sim 10^{\circ}$ with the $\{101\}_{B 2}$ planes. We note that this variant of the wave normals can be used for a combined description of the resultant strain when additional compression in the $\langle\overline{1} 0 \overline{1}\rangle_{B 2}$ directions and tension in the $\langle\overline{1} 1 \overline{1}\rangle_{B 2}$ directions, providing compression of the $\{\overline{1} 1 \overline{1}\}_{B 2}$ planes and the required ratio $\left|\varepsilon^{\|} / \varepsilon^{\perp}\right| \approx 2$, are observed together with the strain induced by the CWP.

1.2. We now find $\boldsymbol{n}_{1}$ and $\boldsymbol{n}_{2}$ for the $(101)_{B 2}$ habit plane given that $\Lambda \|[10 \overline{1}]_{B 2}$. Considering that the $(101)_{B 2}$ plane is intersected with the $(10 \overline{1})_{B 2}$ plane along the $[010]_{B 2}$ line and rotating the $[010]_{B 2}$ vector through the angles $\pm \pi / 4$ about the $[10 \overline{1}]_{B 2}$ axis, we obtain

$$
\boldsymbol{n}_{1} \|[\overline{1} \sqrt{2} 1]_{B 2} \text { and } \boldsymbol{n}_{2} \|[1 \sqrt{2}] \overline{1}_{B 2}
$$

It is clear that for $\Lambda \|[10 \overline{1}]_{B 2}, \boldsymbol{n}_{1}$ and $\boldsymbol{n}_{2}$ can be easily determined formally for the $(h k h)_{B 2}$ habit planes. Analogous CWP variants provide the fastest strain of the $\{10 \overline{1}\}_{B 2}$ planes and incorporation of these planes into the OR.

2. In particular, exact crystallographic orientations of the habit planes $\{011\}_{B 2}$ and $\{001\}_{B 2}$ can be observed, for $\Lambda \|<100>_{B 2}$. These habit planes are specially mentioned, since crystals forming polycrystal ensembles are combined along the above-indicated planes. The CWP corresponding to $\Lambda \|<100>_{B 2}$ provides the fastest transformation of the $\{100\}_{B 2}$ plane and its inclusion in the OR.

3. Let we have $\Lambda \|<111>_{B 2}$.

3.1. As a first example, we choose $\boldsymbol{n}_{2} \|[\overline{1} 2 \overline{1}]_{B 2}$ and $\boldsymbol{n}_{1} \|[10 \overline{1}]_{B 2}$. Then, according to Eq. (2), we obtain 


$$
N_{\mathrm{w}} \|[1 \pm \sqrt{3} \mathfrak{x}, 1 \mp \sqrt{3} \mathfrak{x}, \overline{2}]_{B 2},
$$

and for $æ \approx 1$ (in the approximation of small integer indices) we have the family of the habit planes close to $\{413\}_{B 2}$.

3.2. We now obtain a pair of normals to the $(1 \overline{1} 0)_{B 2}$ habit plane for $\Lambda \|[111]_{B 2}$. We consider that the $(1 \overline{1} 0)_{B 2}$ and $(111)_{B 2}$ planes are intersected along the $\left[\begin{array}{ll}\overline{1} & 1\end{array}\right]_{B 2}$ direction. Then orientations of the normals $\boldsymbol{n}_{1}$ and $\boldsymbol{n}_{2}$ in the

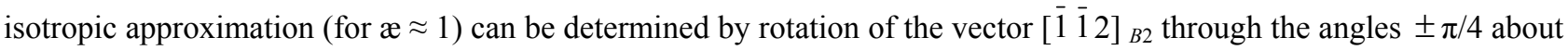
the $[111]_{B 2}$ axis:

$$
\boldsymbol{n}_{1}\left\|[1-\sqrt{3}, 1+\sqrt{3}, \overline{2}]_{B 2}, \boldsymbol{n}_{2}\right\|[1+\sqrt{3}, 1-\sqrt{3}, \overline{2}]_{B 2} .
$$

It can be easily verified that $\left(\boldsymbol{n}_{2}-\boldsymbol{n}_{1}\right) \|[\overline{1} 10]_{B 2}$, that is, it determines the normal to the habit plane. Speaking about the character of the strain transferred by the CWP, it is pertinent to note two extreme cases. First, it is possible to assume that the CWP transfers only the compression strain that causes quasi-static tension (see Section 1) along the $[111]_{B 2}$ direction. It seems likely that this variant is observed in the case of independent formation of individual crystals. The negative volume effect during the transformation, though small, is the evidence in its favor. Second, in the case of the self-consistent formation of polycrystal ensembles, the variants of the CWP transferring strains of opposite signs can be observed, but crystals are combined so that they provide quasi-isotropic resultant strain. In any variant with $\Lambda \|<111>_{B 2}$, the CWP provides the fastest reorganization of the (111) $)_{B 2}$ plane and its inclusion in the OR.

\section{ORIENTATION RELATIONS}

The observed orientation relations, according to [5], are close to the variant

$$
\begin{aligned}
&\{111\}_{B 2} \|(111)_{R}, \\
&< \overline{1} 2 \overline{1}>_{B 2} \|[\overline{1} 2 \overline{1}]_{R}, \\
&<\overline{1} 10>_{B 2} \|[\overline{1} 10]_{R} .
\end{aligned}
$$

As follows from the consideration in Sections 1 and 2, the OR can be interpreted as a consequence of the tension-compression process that preserves the orientation of the $\{111\}_{B 2}$ plane induced by the dynamic strain process of the $\{111\}_{B 2}$ plane upon exposure to the pair of wave beams with wave normals (9). According to [10, 12], it is natural to consider what exactly the plane subject to the fastest strain is involved into the OR, and for the misorientation

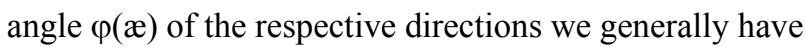

$$
\varphi(\mathfrak{x})=\arccos \frac{\Gamma+\mathfrak{x}^{2}}{\sqrt{\left(\Gamma^{2}+\mathfrak{x}^{2}\right)\left(\mathfrak{x}^{2}+1\right)}}, \Gamma=\frac{1+\varepsilon_{1}}{1-\left|\varepsilon_{2}\right|} .
$$

When the strains are small, from Eq. (11) we derive the approximate relation

$$
\varphi(æ)^{\circ} \approx \frac{180 æ\left(\varepsilon_{1}+\left|\varepsilon_{2}\right|\right)}{\pi\left(æ^{2}+1\right)} .
$$

For the $B 2-R$ MT, it is natural to expect that the CWP provides only small deviations from formulas (10). Indeed, for the strain $\varepsilon_{1} \approx\left|\varepsilon_{2}\right| \approx 0.0764 \%$, we obtain from Eq. (12) the rotation angle of the $<\overline{1} 1 \overline{1}>_{B 2},<121>_{B 2}$ reference point about the $<\overline{1} 01>_{B 2}$ axis $\varphi \approx 0.044^{\circ}$. We note also that for the strain $\varepsilon_{1} \approx\left|\varepsilon_{2}\right| \approx 0.56 \%$ and opposite signs of the 
strains in the CWP field, reference point (9) will rotate about the $<111>_{B 2}$ axis through the angle $\varphi \approx 0.32^{\circ}$. When the strain signs coincide (we must set $\varepsilon_{1}<0$ in Eq. (12)), the angle $\varphi \approx 0^{\circ}$, and in this case, OR (10) hold true.

Thus, the CWP with wave normals (9) transferring equal compression strains in the orthogonal directions and providing the fastest strain of the $\{111\}_{B 2}$ plane corresponds to the observed strain ratio, orientation of the habit plane, and orientation relations.

\section{REFINEMENT OF THE DATA ON THE DISLOCATION NUCLEATION CENTER IN THE APPROXIMATION OF THE ISOTROPIC ELASTIC MEDIUM}

Till now we have not concretized all the details of the dislocation nucleation center. It was suffice to know only the orientation of the dislocation line $\Lambda$. However, it is easy to perform the necessary refinements useful for vivid geometrical interpretation of the morphological parameters. Setting $\Lambda \|<111\rangle_{B 2}$, we now determine the extra plane from the data on the wave normals and the character of the strain. Recall the description of the strain field for the edge orientation of the Burgers vector that determines the sliding plane and is collinear with a normal to the extra plane. It is convenient to describe the strain field of a rectilinear edge dislocation in the infinite isotropic medium in the plane perpendicular to $\Lambda$ using cylindrical coordinates [13]. For a fixed distance $r$ to the line $\Lambda$, the change of the eigenvalues $\varepsilon_{1}$ and $\varepsilon_{2}$ of the strain tensor is characterized by the polar angle $\theta$ counted from the shear vector in the halfspace comprising the extra plane. Here $\theta=-\pi / 2$ corresponds to points of the extra plane. If we do not require that the strain along the direction $\Lambda$ was equal to zero, we obtain

$$
\varepsilon_{1}=\frac{b(\sin \theta+\cos \theta)}{2 \pi r(1-v)}, \varepsilon_{2}=\frac{b(\sin \theta-\cos \theta)}{2 \pi r(1-v)},
$$

where $b$ is the modulus of the Burgers vector $\boldsymbol{b}$ having the edge orientation. The normalized eigenvectors of the strain tensor $\xi_{i}$ determined by projections onto the axes $x_{1}\left\|\boldsymbol{b}, x_{2}\right\|[\Lambda \boldsymbol{b}]$, and $x_{3} \| \Lambda$ have the form

$$
\begin{gathered}
\xi_{1}=\left[\begin{array}{lll}
\cos \left(\theta-\frac{\pi}{4}\right) & \sin \left(\theta-\frac{\pi}{4}\right) & 0
\end{array}\right], \\
\xi_{2}=\left[\begin{array}{lll}
-\sin \left(\theta-\frac{\pi}{4}\right) & \cos \left(\theta-\frac{\pi}{4}\right) & 0
\end{array}\right], \\
\xi_{3}=\left[\begin{array}{lll}
0 & 0 & 1
\end{array}\right] .
\end{gathered}
$$

If we require that the strain along the direction $\Lambda$ was equal to zero, the eigenvalues must be renormalized, and instead of Eq. (13), we obtain

$$
\varepsilon_{1}=\frac{b((1-2 v) \sin \theta+\cos \theta)}{4 \pi r(1-v)}, \varepsilon_{2}=\frac{b((1-2 v) \sin \theta-\cos \theta)}{4 \pi r(1-v)} .
$$

From Eqs. (13) and (15) it follows that the equal negative values $\varepsilon_{1}=\varepsilon_{2}<0$ are observed at $\theta=-\pi / 2$ in the compression field related to the extra plane. From Eq. (14) it follows that at $\theta=-\pi / 2$, the eigenvectors of the strain tensor are at angles of $\pm \pi / 4$ to the extra plane. This means that the extra plane for normals (9) coincides with the orientation of the habit plane. For vectors $\boldsymbol{b} \|<100>_{B 2}$ typical of the $B 2$ phase, dislocations with lines $\Lambda \|<111>_{B 2}$ are mixed. Hence, the eigenvectors $\xi_{1}$ and $\xi_{2}$ (and hence the wave normals) deflect from the $\{111\}_{B 2}$ planes. Recall that for a purely screw dislocation $(\Lambda \| \boldsymbol{b})$ in the isotropic medium, one of the eigenaxes (for the eigenvalue equal to zero) coincides with the radial direction in the plane perpendicular to $\Lambda$, and two others (tension and compression axes) form the angle $\pi / 4$ with this plane for any arbitrary $\theta$ (the tension-compression plane is parallel to $\Lambda$ ). Therefore, in general 
24 variants of the habit planes can be observed rather than six variants (in the degenerate case) when the normals to the habit plane lie in the stereographic orientation triangle. Accordingly, four poles of the normals to the habit planes are grouped in the vicinity of each pole $\langle 110\rangle_{B 2}$. Three extra planes (close to the habit planes) and hence six habit planes are matched with each line $\Lambda$. The observed accommodation variants of forming packet-pyramidal morphology of crystal ensembles with the $R$-phase were described in detail in Section 3.4.2 of monograph [5].

\section{CONCLUSIONS}

Our analysis has demonstrated that the morphological parameters observed during the $B 2-R$ MT within the framework of the dynamic theory [1-4] can provide adequate treatment, despite the proximity of the transformation to the second-order transitions. Small final strains require precise measurements of the OR to compare them with calculated values. When the accuracy of measuring the OR reached $0.01^{\circ}$, the calculated misorientation planes and the corresponding directions included into the OR must be refined considering though small, but still nonzero anisotropy of the elastic properties. In addition, it is clear that taking the anisotropy into account, the fastest strain of the $\{111\}_{B 2}$ plane induced by the CWP cannot be attributed to the DNC having the edge dislocation whose line is oriented strictly along the third-order $\langle 111\rangle_{B 2}$ symmetry axis as a basic segment. This was analyzed in detail in [15].

This work was supported in part by the Russian Foundation for Basic Research (project No. 11-08-96020).

\section{REFERENCES}

1. M. P. Kashchenko and V. G. Chashchina, Physics-Uspekhi, 54, No. 4, 331-349 (2011).

2. M. P. Kashchenko and V. G. Chashchina, Pis'ma Mater., 1, 7-15 (2011).

3. M. P. Kashchenko and V. G. Chashchina, Mater. Sci. Forum, 738-739, 3-9 (2013).

4. M. P. Kaschenko, Wave Model of Martensite Growth during $\gamma-\alpha$ Transformation in Iron-Based Alloys [in Russian], Scientific and Publishing Center "Regular and Chaotic Dynamics," Institute of Computer Sciences, Moscow; Izhevsk (2010).

5. Titanium Nickelide Alloys with Shape Memory. Part I. Structure, Phase Transformations, and Properties [in Russian], Publishing House of the Ural Branch of the Russian Academy of Sciences, Ekaterinburg (2006).

6. V. G. Pushin, V. V. Kondrat'ev, and V. N. Khachin, Pre-transitive Phenomena and Martensitic Transformations [in Russian], Publishing House of the Ural Branch of the Russian Academy of Sciences, Ekaterinburg (1998).

7. I. V. Alexina, N. V. Aristova, and M. P. Kashchenko, in: Shape Memory, International Academic Publishers, Beijing (1994), pp. 65-69.

8. M. P. Kashchenko, I. V. Alexina, V. V. Letuchev, and A. V. Nefedov, Fiz. Met. Metallogr., 80, No. 6, 10-15 (1995).

9. M. P. Kashchenko and V. G. Chashchina, Fiz. Met. Metallogr., 105, No. 6, 537-543 (2008).

10. M. P. Kashchenko and V. G. Chashchina, Fiz. Met. Metallogr., 106, No. 1, 14-23 (2008).

11. A. A. Il'in, Mechanism and Kinetics of Phase and Structural Transformations in Titanium Alloys [in Russian], Nauka, Moscow (1994).

12. M. Kashchenko and V. Chashchina, Dynamic Theory of $\gamma-\alpha$ Martensitic Transformation in Iron-Based Alloys. Solving the Problem of the Formation of Twinned Martensite Crystals, LAMBERT Academic Publishing, Saarbrucken (2012).

13. J. Hirt and I. Lote, Teory of Dislocations [Russian translation], Atomizdat, Moscow (1972).

14. M. P. Kashchenko and V. P. Vereshchagin, Sov. Phys. J., 32, No. 8, 592-595 (1989).

15. M. P. Kashchenko, K. N. Dzhemilev, and V. G. Chashchina, Fund. Probl. Sovrem. Materialoved., 9, No. 1, 50-56 (2012). 